\title{
Учебно-методическая работа
}

УДК 621.9.014

\section{Методика проектирования сборных токарных резцов, оснащенных СМП с нулевым задним углом}

\author{
Б.Д. Даниленко \\ МГТУ им. Н.Э. Баумана, 105005, Москва, Российская Федерация, 2-я Бауманская ул., д. 5, стр. 1.
}

\section{An approach to design modular carbide-tipped cutting tools with zero back angles}

\author{
B.D. Danilenko \\ Bauman Moscow State Technical University, building 1, 2-nd Baumanskaya, 5, 105005, Moscow, Russian Federation. \\ e-mail: danilenko@bmstu.ru
}

\begin{abstract}
В настоящее время большинство твердосплавных инструментов оснащаются сменными многогранными пластинками (СМП), а для получения оптимальной геометрии лезвия пластинки должны быть ориентированы в корпусе инструмента строго определенным образом. В статье предложен научно обоснованный графо-аналитический расчет параметров установки СМП. Новизна данной методики заключается в том, что она в определенной степени универсальна и может быть использована при проектировании других инструментов, оснащенных СМП. Приведено описание порядка расчета конструктивных параметров державки сборного токарного резца, оснащенного СМП с нулевым задним углом. Полученные в результате расчета параметры конструкции обеспечивают оптимальность геометрических характеристик лезвия резца. Методика анализа получаемых геометрических параметров может служить основой для анализа оптимальности конструкции инструмента, в том числе, при выполнении студенческих научных работ.
\end{abstract}

Ключевые слова: державка резца, задний угол, оптимальная геометрия, графоаналитический расчет, параметры установки.

Most cutting tools are now carbide tipped. To obtain the optimum geometry, the removable faceted cutting tips (RFTs) must be oriented in a certain way relative to the tool. This paper proposes a scientifically based graphical-analytical approach to calculate the RCT parameters. The proposed approach is universal to some extent and can be used for designing other tools equipped with RFTs. An algorithm for calculating the design parameters of a modular lathe tool bit holder with RFTs and a zero back angle is described. The calculated design parameters provide the optimal geometry of the cutting tool edge. The analysis of the derived geometric parameters can form the basis for the optimal design of cutting tools, which can be the topic of students' research.

Keywords: tool holder, rear angle, optimal geometry, graphical-analytical approach, design parameters.

В настоящее время большинство режущих инструментов с режущей частью из твердого сплава оснащаются сменными многогранными пластинками (СМП) $[1,2]$. Для получения оптимальных геометрических параметров режущего лезвия СМП должна быть установлена в корпусе инструмента строго определенным образом [3]. Положение пластинки зависит от количества режущих граней СМП, формы передней поверхности (плоская или с углублениями для получения положительного пере- 
днего угла), наличия на пластинке заднего угла и других факторов.

Пространственное расположение опорной плоскости под СМП задается углами наклона в статической системе координат [4].

Рассмотрим порядок расчета установочных углов для опорной плоскости СМП с плоской передней поверхностью и без заднего угла $\alpha$ [5]. Угол $\alpha$ обеспечивается установкой СМП на державке с образованием отрицательного переднего угла. Положение пластинки должно обеспечивать работоспособное состояние каждой режущей кромки и наибольший период стойкости резца при данных условиях и режиме точения.

Задача оптимизации геометрических параметров в этом случае является более сложной, чем у резца с напаянной пластинкой, где каждая из граней затачивается отдельно и углам режущей кромки придается оптимальная величина. Действительно, ориентация пластинки данной формы, выполненная так, чтобы образовать оптимальный задний угол $\alpha$ на главной кромке, одновременно обусловливает величины всех

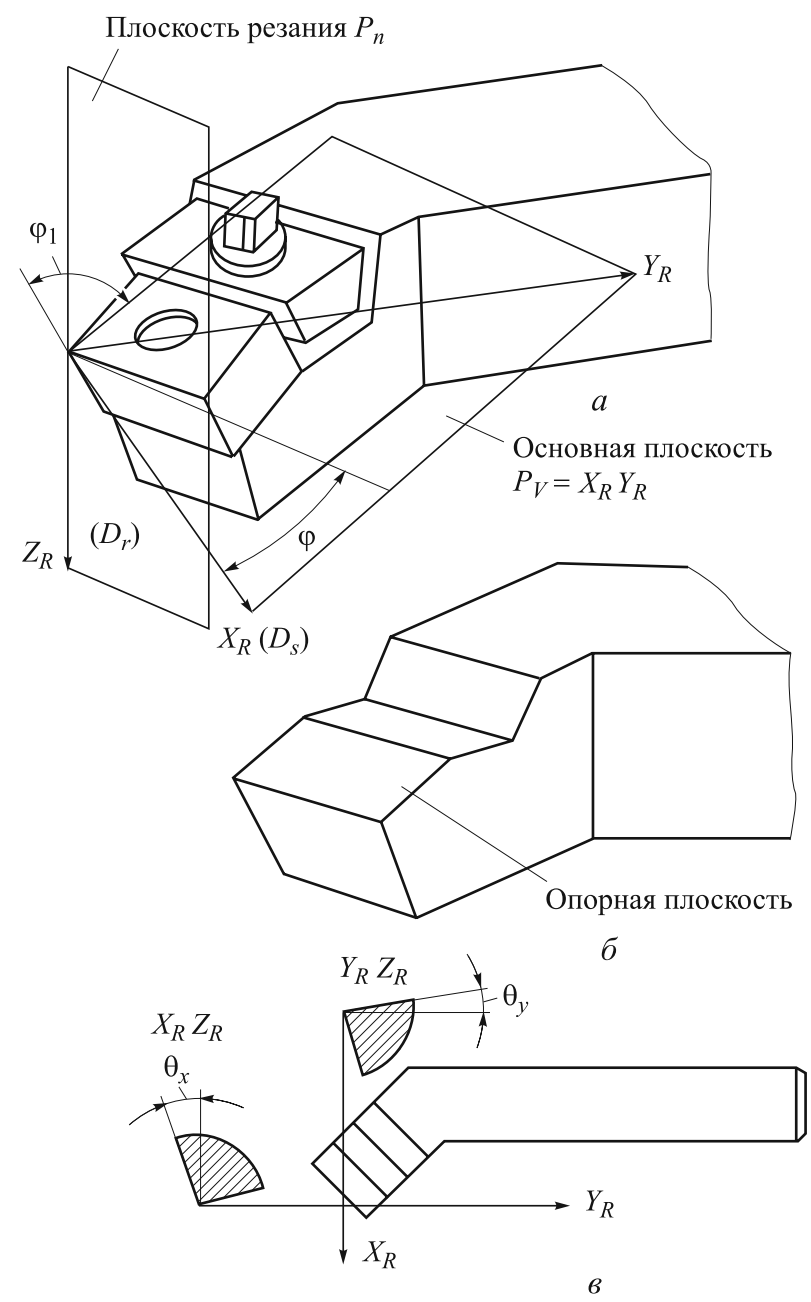

Puc. 1. Пример проектируемого резца: $a$ - расположение СМП на державке резца; 6 - опорная плоскость под СМП; 6 - углы ориентации опорной плоскости остальных углов резца, причем они могут существенно отличаться от остальных.

Резец с механическим креплением квадратной СМП показан на рис. $1, a$, а корпус резца без СМП (отверстия не показаны) - на рис. 1, 6, в. Для получения оптимальной геометрии резца опорная плоскость СМП должна быть ориентирована определенным образом относительно осей координат $X_{R}, Y_{R}$, $Z_{R}$. Это положение опорной плоскости определяется наклоном в плоскости $Y_{R} Z_{R}-$ углом $\theta_{Y}$, а в плоскости $X_{R} Z_{R}$ - углом $\theta_{X}$ (см. рис. 1,8$)$. Для решения оптимизационной задачи необходимо установить:

1) параметры ориентации пластины относительно статической системы координат резца, т. е. положение опорной плоскости, характеризуемое углами $\theta_{X}$ и $\theta_{Y}$;

2) аналитические зависимости между геометрическими параметрами СМП и резца с установленной СМП и параметрами ориентации СМП;

3) значения основных геометрических параметров резца при данных параметрах СМП и параметрах ориентации;

4) вычисленные геометрические параметры лежат в допустимых пределах.

Расчетная схема параметров СМП приведена на рис. 2. Здесь режущая СМП представлена одной из ее вершин, причем радиус скругления вершины условно принят равным нулю. Статическая система координат резца $X_{R} Y_{R} Z_{R}$ имеет начало $O$ в вершине пластины, a ее ось $X_{R}$ совпадает с направлением подачи $D_{s}$ (см. рис. 1, $a)$.

Для получения положительного заднего угла (на главной кромке необходимо повернуть СМП вокруг оси (например, оси $X$ ) на угол $\theta$. Чтобы угол $\theta$ на расчетной схеме проецировался в натуральную величину, вводится новая система координат $X Y Z$, которая получается путем поворота прежней оси вокруг оси $Z$ против часовой стрелки на угол $\mu$. Таким образом, параметрами ориентации пластины являются следующие:

1) угол $\mu$, характеризующий положение оси поворота относительно прежней системы координат $X_{R} Y_{R} Z_{R}$, и положение новой системы координат $X Y Z$ относительно прежней $X_{R} Y_{R} Z_{R}$;

2) угол $\theta$ поворота СМП вокруг оси $X$ против часовой стрелки.

Параметры ориентации - углы $\mu$ и $\theta-$ позволяют управлять величинами задних углов $\alpha$ и $\alpha_{1}$.

Для построения расчетной схемы и вычисления геометрических параметров необходимо задаться следующими значениями:

1) заднего угла $\alpha$. Оптимальная величина заднего угла зависит от принятых режимов резания. Будем считать, что задний угол $\alpha$ может выбираться в пределах $6 \ldots 15^{\circ}$;

2) главного угла в плане $\varphi$. Выбор угла $\varphi$ определяется типом операции (чистовая, черновая), жесткостью заготовки, конструкцией державки и пластины и т. п. Для условий данного расчета выберем угол $\varphi$ в пределах $30 \ldots 75^{\circ}$;

3) угла расположения оси поворота пластины $\mu$. Этот угол в значительной мере определяется углом при вершине пластины $\varepsilon_{0}$ и $\varphi$. 


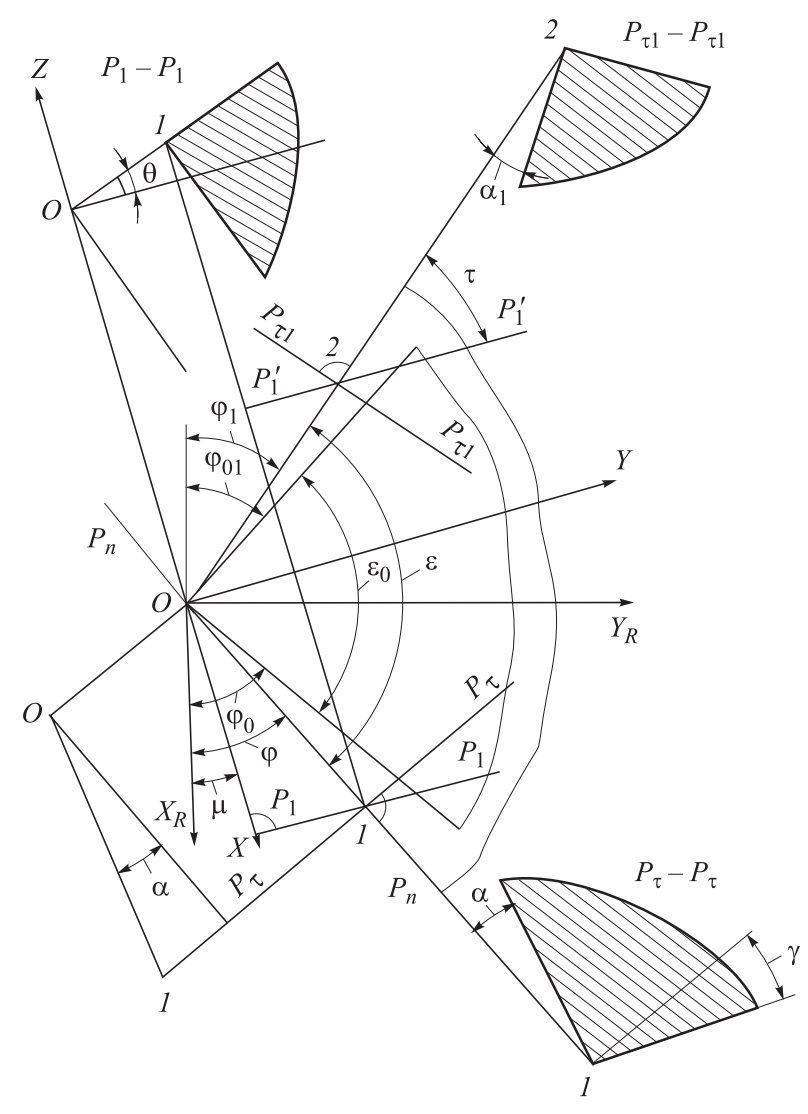

Puc. 2. Расчетная схема определения геометрических параметров СМП

Так, например, если расположить ось поворота $X$ перпендикулярно биссектрисе угла $\varepsilon$, что примерно соответствует $\mu=\varphi-(\pi-\varepsilon) / 2$, и повернуть СМП на угол $\theta$, то задние углы главной и вспомогательной кромок будут равными, что обычно используется для резцов напайного типа. Для сборного резца с многогранной СМП такое положение можно получить только в частном случае. Естественно, более важно обеспечить необходимую величину угла $\alpha$.

При увеличении угла $\mu$ на $\Delta \mu$ и постоянном угле поворота $\theta$ величины задних углов изменятся: $\alpha$ будет увеличиваться, а $\alpha_{1}-$ уменьшаться. Для условий данного расчета угол $\mu$ в первом приближении будем выбирать несколько большим значения, которое определяется по формуле $\mu=\varphi-\left(\pi-\varepsilon_{0}\right) / 2$. Это позволит обеспечить правильное соотношение $\alpha \geq \alpha_{1}>1 . .1,5^{\circ}$. Для черновой обработки лучше использовать $\alpha=\alpha_{1}$, а для чистовой обработки величина угла $\alpha_{1}$ не имеет большого значения, и можно принимать $\alpha_{1}=1 \ldots 1,5^{\circ}$.

Построение схемы начинается с проекции СМП на основную плоскость $P_{v}\left(X_{R} Y_{R}\right.$, см. рис. 2). Начало $O$ статической системы координат резца совпадает с вершиной режущей пластины, а ось $X_{R}-$ с направлением подачи. Под углом $\varphi$ проводится след плоскости резания $P_{n}$, совпадающий с проекцией главной режущей кромки на основную плоскость (см. рис. $1, a)$. Строится сечение главной секущей плоскостью $P_{\tau}$ в точке 1 и в сечении $P_{\tau}-P_{\tau}$ проводится след задней поверхности под углом $\alpha$.
После определения направления оси поворота $X$ необходимо определить угол $\mu$. Этот угол в первом приближении будем устанавливать примерно на $5^{\circ}$ больше той величины, которая получается по формуле, т. е. $\mu=\varphi-\left(\pi-\varepsilon_{0}\right) / 2+5^{\circ}$.

Через точку 1 проводим след секущей плоскости $P_{1}-P_{1}$, перпендикулярной оси поворота $X$, где угол $\theta$ будет проецироваться в натуральную величину. Для построения сечения $P_{1}-P_{1}$ необходимо рассчитать значение угла $\theta$ по формуле (1). Затем вычисляется передний угол $\gamma$ по формуле (2) и проводится след передней поверхности в сечении $P_{\tau}-P_{\tau}$.

Исходный угол в плане $\varphi_{0}$ определяется по формуле (3), затем находится исходное положение главной кромки СМП. Исходное положение вспомогательной кромки, определяемое углом $\varphi_{01}$, определяется графически после расчета угла $\varphi_{01}$ по формуле (4) или через $\varepsilon_{0}$.

Далее по формуле (5) вычисляется угол $\tau$, который определяет положение проекции вспомогательной кромки на основную плоскость $P_{v}\left(X_{R} Y_{R}\right)$ по отношению к следу плоскости, перпендикулярной к оси поворота $P_{1}^{\prime} P_{1}^{\prime}$. Затем по формуле (6) определяется угол $\varphi_{1}$, и на схеме дается проекция вспомогательной кромки на основную плоскость. После определения вспомогательного заднего угла $\varphi_{1}$ по формуле (7) на схеме строится сечение $P_{\tau}-P_{\tau}$, причем след передней поверхности в этом сечении можно провести произвольно. По формуле (8) рассчитывается угол наклона главной кромки $l$.

Если числовые значения главных геометрических параметров лежат в допустимых пределах, по формулам (9) и (10) вычисляются технологические углы $\theta_{Y}$ и $\theta_{X}$, которые используются как установочные для операции фрезерования опорной плоскости или гнезда под СМП.

Расчетные формулы [1]:

$$
\begin{gathered}
\operatorname{tg} \theta=\frac{\cos (\varphi-\mu)-\sqrt{\cos ^{2}(\varphi-\mu)-4 \operatorname{tg}^{2} \alpha \sin ^{2}(\varphi-\mu)}}{2 \operatorname{tg} \alpha \sin ^{2}(\varphi-\mu)} ; \\
\operatorname{tg} \gamma=\operatorname{tg} \theta \cos (\varphi-\mu),
\end{gathered}
$$

угол $\gamma$ - отрицательный;

$$
\operatorname{tg}\left(\varphi_{0}-\mu\right)=\frac{\operatorname{tg}(\varphi-\mu)}{\cos \theta}
$$

Отсюда определяется $\varphi_{0}$;

$$
\begin{gathered}
\varphi_{01}=\pi-\varepsilon_{0}-\varphi_{0} ; \\
\operatorname{tg} \tau=\frac{\operatorname{tg}\left(\frac{\pi}{2}-\varphi_{01}-\mu\right)}{\cos \theta} ; \\
\varphi_{1}=\frac{\pi}{2}-\mu-\tau ; \\
\operatorname{tg} \alpha_{1}=\frac{\sin \tau \operatorname{tg} \theta}{1+\cos ^{2} \tau \operatorname{tg}^{2} \theta} ; \\
\operatorname{tg} \alpha=\operatorname{tg} \theta \sin (\varphi-\mu) ;
\end{gathered}
$$


$\operatorname{tg} \theta_{Y}=\operatorname{tg} \theta \sin \mu$

$\operatorname{tg} \theta_{X}=\operatorname{tg} \theta \cos \mu$
Статья может быть использована в качестве руководства для выполнения студентами расчетов и конструирования инструмента, оснащенного СМП.

\section{Литература}

[1] Верещака А.С., Кушнер В.С. Резание материалов. Москва, Высшая школа, 2009. 535 с.

[2] Каталоги фирмы Sandvik Coromant 2000-2012.

[3] Петрушин С.И., Грубый С.В. Обработка чугунов и сталей сборными резиами со сменными многогранными пластинами. Томск, Изд-во ТПУ, 2000. 156 с.

[4] Розенберг Ю.А. Резание материалов. Курган, Изд-во ОАО ПК «Зауралье», 2007. 294 с.

[5] Дальский А.М., ред. Справочник технолога-машиностроителя. В 2 т. Т. 2. Москва, Машиностроение, 2001. 944 c.

\section{References}

[1] Vereshchaka A.S., Kushner V.S. Rezanie materialov [Cutting of materials]. Moscow, Vysshaia shkola publ., 2009. 535 p.

[2] Katalogi firmy Sandvik Coromant 2000-2012 [Catalogues firm Sandvik Coromant 2000-2012].

[3] Petrushin S.I., Grubyi S.V. Obrabotka chugunov i stalei sbornymi reztsami so smennymi mnogogrannymi plastinami [Processing of cast iron and steel cutters teams with indexable inserts]. Tomsk, TPU publ., 2000. 156 p.

[4] Rozenberg Iu.A. Rezanie materialov [Cutting of materials]. Kurgan, Zaural'e publ., 2007. 294 p.

[5] Spravochnik tekhnologa-mashinostroitelia [Directory technologist-machinist]. Ed. Dal'skii A.M. In 2 vol., vol. 2. Moscow, Mashinostroenie publ., 2001. 944 p.

Статья поступила в редакцию 23.12.2013

\section{Информация об авторе}

ДАНИЛЕНКО Борис Дмитриевич (Москва) - кандидат технических наук, доцент кафедры «Инструментальная техника и технология». МГТУ им. Н.Э. Баумана (105005, Москва, Российская Федерация, 2-я Бауманская ул., д. 5, cтр. 1, e-mail: danilenko@bmstu.ru).

\section{Information about the author}

DANILENKO Boris Dmitrievich (Moscow) - Cand. Sc. (Eng.), Associate Professor of «Tool Equipment and Technologies» Department. Bauman Moscow State Technical University (BMSTU, building 1, 2-nd Baumanskaya str., 5, 105005, Moscow, Russian Federation, e-mail: danilenko@bmstu.ru).

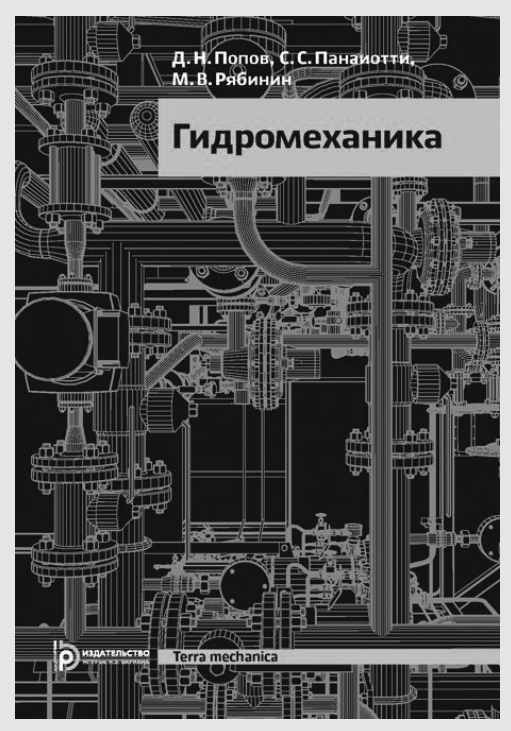

В Издательстве МГТУ им. Н.Э. Баумана вышло в свет учебное пособие

\section{«Гидромеханика»}

авторов Д.Н. Попова, С.С. Панаиотти, М.В. Рябинина.

Изложены основы механики жидкости и газа, а также их приложения в расчетах машин, приводов и других технических устройств. Наряду с традиционными разделами курса гидромеханики рассмотрены актуальные вопросы неустановившегося движения.

По вопросам приобретения обращайтесь:

105005, Москва, 2-я Бауманская ул., д. 5, стр. 1. Тел.: +7 499 263-60-45, факс: +7 499 261-45-97; press@bmstu.ru; www.baumanpress.ru 